\title{
Multifunctional Lymph-Targeted ZnO:Gd@Hyaluronic Acid Nanospheres: Preparation and Application in Tumor Diagnose
}

\author{
Jiejun Cheng, ${ }^{\dagger, a}$ Huajuan Zhang, ${ }^{\dagger, b}$ Jiong Zhu, ${ }^{a}$ Lijun Qian, ${ }^{a}$ Qing Lu, ${ }^{a}$ Guangyu Wu, ${ }^{a}$ \\ Jianrong $\mathrm{Xu},{ }^{,}, a$ and Jun Zhu, ${ }^{\star, b}$ \\ ${ }^{a}$ Department of Radiology, Shanghai Renji Hospital, Shanghai Jiao Tong University School of Medicine, \\ Shanghai 200127, China \\ ${ }^{b}$ National Engineering Research Center for Nanotechnology, Shanghai 200241, China
}

\begin{abstract}
A novel multi-modal lymph-targeted contrast agent, ZnO:Gd@hyaluronic acid (HA) nanospheres, is prepared by Gd dopped ZnO quantum dot, modified by silica shell and grafted by HA molecule. The physicochemical properties are characterized and the lymphatic targeting is evaluated in vitro. Moreover, the contrast agent is used for in vivo lymphoma magnetic resonance imaging (MRI). The results show that the ZnO:Gd@HA nanospheres with 19\% of quantum yield and $5.8 \mathrm{mM}^{-1} \cdot \mathrm{s}^{-1}$ of relaxivity could be used for in vivo lymphatic targeting studies. Furthermore, they are highly targeted to lymph system in vitro and in vivo, and highlight their MRI in lymph tumor. Thus, the novel MRI contrast agent can be taken up selectively by lymphatic system and used as a potential multi-modal contrast agent in lymphatic system.
\end{abstract}

Keywords multi-modal imaging, lymph-targeted, hyaluronic acid, quantum dot

\section{Introduction}

Recently, multi-modal imaging techniques have received considerable attention since they provide a wealth of accurate and detail information for biomedical applications. Among them, magnetic resonance imaging (MRI) is one of the most powerful techniques because of their noninvasive diagnosis, deep penetration into tissues and harmlessness. However, there are some drawbacks, including low sensitivity, spatial resolution, and so on. ${ }^{[1]}$ In contrast, fluorescence imaging offers much higher sensitivity and spatial resolution, but limited depth perception. ${ }^{[2]}$ Therefore, it is a promising approach to integrate the complementary techniques into one platform.

The preparation and application of multifunctional inorganic nanoparticles incorporating magnetic and fluorescent materials has become more and more popular. ${ }^{[3]}$ Some cadmium dichalcogenide nanocomposites have been focused because of their high quantum yield (QY) or relaxivity $\left(r_{1}\right),{ }^{[4,5]}$ but their disadvantages are the high toxicity due to the releasing of $\mathrm{Cd}^{2+}$ or/and $\mathrm{Se}^{2+}$, which limits their biomedical applications. ${ }^{[6,7]}$ Therefore, several biocompatible nanomaterials have been designed to obtain multifunctional contrast agents. ${ }^{[8-10]}$ As a kind of versatile materials, $\mathrm{ZnO}$ was cheap and biocompatible. ${ }^{[11]}$ Various photoluminescent
$\mathrm{ZnO}$ nanoparticles have been investigated and demonstrated their promising biomedical applications. ${ }^{[12]}$ Furthermore, some multi-modal imaging nanoprobes based on $\mathrm{ZnO}$ have been developed. ${ }^{[13,14]}$ However, these nanoprobes rarely results from $\mathrm{ZnO}$ quantum dots with higher QY except there were few reports on Gd-doped $\mathrm{ZnO}$ nanoparticles. For example, Liu et al. ${ }^{[15]}$ fabricated Gd-doped $\mathrm{ZnO}$ quantum dots coated by silica. These biocompatible products exhibited both strong positive contrast effects with $r_{1}$ value of $16 \mathrm{mM}^{-1} \cdot \mathrm{s}^{-1}$ and fluorescence intensity with QYs from $13.5 \%$ to $34 \%$. Most importantly, they proved that Gd doping could enhance the fluorescence intensity of $\mathrm{ZnO}$ quantum dots when the Gd doping ratio is 0.08 . Though the exact mechanism of this phenomenon is not clear, some researchers attributed this luminescence enhancement to less excited states and weak excited state absorption of $\mathrm{Gd}^{3+}$, which led to emission intensity enhancement by effectively energy tranfer. ${ }^{[16]}$

As well known, tumor lymph metastasis is one of the most important reasons causing death. Compared to traditional lymph node biopsy for the diagnosis of metastatic lymph nodes, non-invasive imaging techniques play a great role. Thus, lymph system targeted contrast agent is attracting more and more interest. ${ }^{[17-19]}$ However, most of reported contrast agents for lymph metastasis are based on the mechanism of passive targeting,

\footnotetext{
* E-mail: xujianr@hotmail.com, yzjzhu@163.com

Received December 10, 2015; accepted January 18, 2016; published online January 27, 2016.

${ }^{\dagger}$ These authors contributed equally to this work.
} 
which brings that their imaging results could be affected by the number and function of macrophages in the lymph nodes and finally estimated wrongly by clinical doctors. ${ }^{[20,21]}$ Therefore, developing new targeted contrast agent for lymph metastasis is beneficial to the study and diagnosis of the tumor lymph node metastases, which could bring fresh prospect for the tumor treatment and vast their future development. Because of the interaction between hyaluronic acid (HA) and the CD44 receptor, HA is widely used as a tumor vector for the targeting procedure. CD44 is expressed in a variety of tumors such as breast, colon, intestinal, and brain. ${ }^{[22,23]}$ Furthermore, LYVE-1, as a new homologue of the CD44 glycoprotein, is a lymph-specific receptor for HA, which can bind both soluble and immobilized HA. However, unlike CD44, the LYVE-1 molecule can colocalize with HA on the luminal face of the lymph vessel wall and is completely absent from blood vessels. ${ }^{[24]}$ Therefore, HA is a highly promising lymphatic targeting carrier.

In this paper, we develop a novel multi-modal contrast agent of ZnO:Gd@HA nanospheres based on Gd dopped $\mathrm{ZnO}$ quantum dot, perform their magnetic resonance (MR) and fluorescence imaging in vitro, present evidence that they are highly targeted to lymph system in vitro and in vivo, and highlight their MRI in lymph tumor. Therefore, the contrast agent is capable of defining the multi-modal imaging about lymph tumor, which has great potential in biological area.

\section{Experimental}

\section{Preparation of $\mathrm{ZnO}$ :Gd quantum dots}

$\mathrm{ZnO}$ :Gd quantum dots were prepared according to the following processes with slight modifications. ${ }^{[25]}$ Methacrylic acid (MAA) was mixed with deionized water by $V($ MAA $): V\left(\mathrm{H}_{2} \mathrm{O}\right)=1: 4$, which was heated to $60{ }^{\circ} \mathrm{C}$ and then reacted with appropriate amount of $\mathrm{ZnO}$ powder under stirring for $1 \mathrm{~h}$ to get zinc methacrylate $\left[\mathrm{Zn}(\mathrm{MAA})_{2}\right]$ aqueous solution. The unreacted $\mathrm{ZnO}$ powder was removed with filtration. The $\mathrm{Zn}(\mathrm{MAA})_{2}$ aqueous solution was dehydrated in a freeze drier at $-80{ }^{\circ} \mathrm{C}$ to obtain anhydrous $\mathrm{Zn}(\mathrm{MAA})_{2}$ powder. Furthermore, $10 \mathrm{mmol}$ of anhydrous $\mathrm{Zn}(\mathrm{MAA})_{2}$ powder and $0.8 \mathrm{mmol}$ of $\mathrm{Gd}\left(\mathrm{NO}_{3}\right)_{3} \cdot 6 \mathrm{H}_{2} \mathrm{O}$ were dissolved in 100 $\mathrm{mL}$ of absolute ethanol, which was refluxed for $1 \mathrm{~h}$ at $80{ }^{\circ} \mathrm{C}$. Finally, $153 \mathrm{mmol}$ of $\mathrm{LiOH} \cdot \mathrm{H}_{2} \mathrm{O}$ ethanol solution, a certain content of $\mathrm{LiOH} \cdot \mathrm{H}_{2} \mathrm{O}$ dissolved in 100 $\mathrm{mL}$ of absolute ethanol by sonication, was added into the above mixture solution. After stirring for $24 \mathrm{~h}$ at room temperature, the as-prepared products were separated by centrifugation and then dispersed in $200 \mathrm{~mL}$ of ethanol or cyclohexane for further experiments.

\section{Preparation of ZnO:Gd@silica nanoparticles}

The ZnO:Gd@silica nanoparticles were prepared as follows: $7.5 \mathrm{~mL}$ cyclohexane of as-prepared $\mathrm{ZnO}$ :Gd nanoparticles, 1.77 mL Triton X-100, $1.8 \mathrm{~mL} n$-hexanol,
$48 \mu \mathrm{L}$ deionized water and $240 \mu \mathrm{L}$ ammonia aqueous solution (25 wt\%) were mixed under stirring to form microemulsion system. After $30 \mathrm{~min}, 100 \mu \mathrm{L}$ tetraethyl orthosilicate (TEOS) was added and reacted for $24 \mathrm{~h}$, and then $50 \mu \mathrm{L} \gamma$-aminopropyltriethoxysilane (APS) was introduced. The reaction was continued for another $12 \mathrm{~h}$ at room temperature. The resulting ZnO:Gd@silica nanoparticles were collected by centrifugation.

\section{Preparation of ZnO:Gd@HA nanospheres}

HA was activated using $N$-hydroxysuccinimide (NHS) and $N$-ethyl- $N$ '-[3-(dimethylamino)propyl] carbodiimide hydrochloride (EDC). Specially, $1.25 \mathrm{~g}$ of HA was dissolved in $200 \mathrm{~mL}$ double-distilled water. Subsequently, $0.23 \mathrm{~g}$ of EDC and $0.14 \mathrm{~g}$ of NHS were added into the solution and 0.1 g of ZnO:Gd@silica nanoparticles was dispersed in the solution. The mixed solution was stirred for $24 \mathrm{~h}$ at room temperature. Finally, the resulting products of ZnO:Gd@HA nanospheres were collected by centrifuging, washing with ethanol and water three times, and drying at $60{ }^{\circ} \mathrm{C}$ in sequence.

\section{Characterization}

Powder X-ray diffraction (XRD) patterns were recorded on a Rigaku D/MAX-2250V diffractometer using Cu-Karadiation (40 kV and $40 \mathrm{~mA}$ ). The morphology of the samples was obtained using a high resolution transmission electron microscope (HRTEM, JEOL, JEM-2100F) and a field-emission scanning electron microscope (FESEM, Hitachi, S-4800), respectively. The Fourier transformation infrared (FT-IR) spectra were recorded on a Nicolet 6700 FT-IR spectrometer. The UV-vis spectra were investigated using a PerkinElmer Lambda 950 UV-vis spectrophotometer. Photoluminescence spectra were recorded using a Hitachi fluorescence spectrophotometer (F-7000).

\section{Confocal imaging of cells}

Confocal imaging of cells was performed using a Leica laser scanning confocal microscope. HCT116 cells $\left(1 \times 10^{6}\right.$ cells $\left./ \mathrm{mL}\right)$ were incubated with ZnO:Gd@ HA nanospheres for $2 \mathrm{~h}$ for confocal imaging, fixed with $4 \%$ paraformaldehyde for $30 \mathrm{~min}$ and stained by 2-(4-amidinophenyl)-6-indolecarbamidine dihydrochloride (DAPI) for $8 \mathrm{~min}$. All the cells were washed twice with PBS before confocal imaging. Imaging of ZnO:Gd@HA nanospheres was carried out at 405 nm laser excitation, with its emission collected from 500 to $550 \mathrm{~nm}$.

\section{Measurement of MRI signal}

All MRI signals were carried out with a $3.0 \mathrm{~T}$ whole body system (3.0 T Intera Achieva, Philips Medical Systems, Best, The Netherlands) with brain Crossed Coil. Different mass concentrations of ZnO:Gd@HA nanospheres were placed in a series of PE tubes for $T_{1}$-weighted MR imaging, using a standard spin echo 


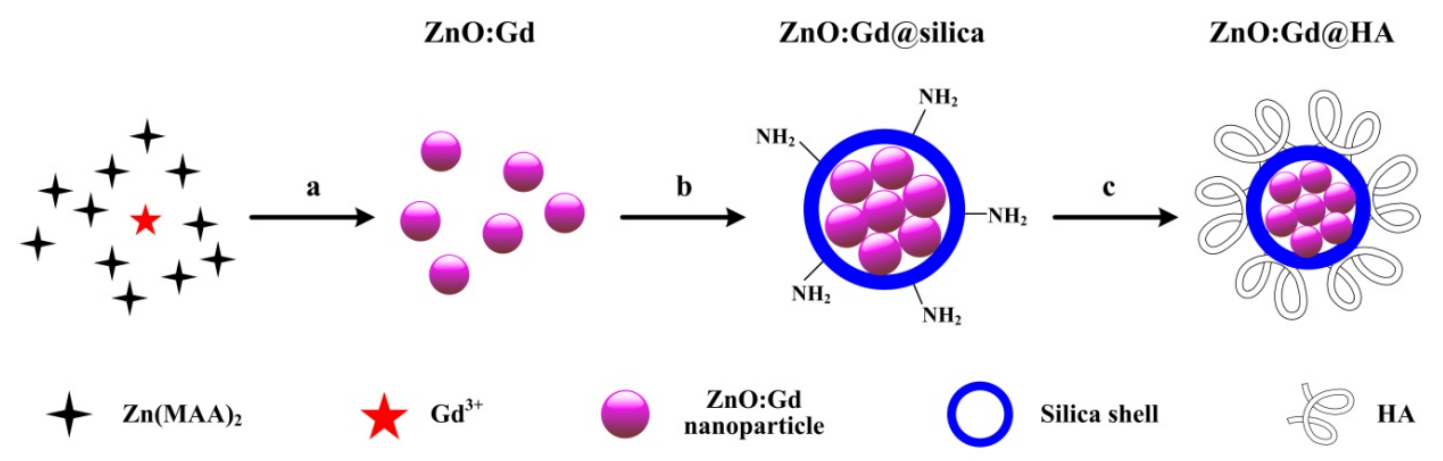

Figure 1 Schematic illustration of the formation of $\mathrm{ZnO}$ :Gd@HA nanospheres.

sequence. The sequence parameters were TR/TE, 4.6/2.4 ms; flip angle, 12 ; FOV, $26 \mathrm{~cm}$; matrix, $320 \times$ 256; effective slice thickness, $0.6 \mathrm{~mm}$. The 3D MRIs were then reconstructed from the original data set at each time point using maximum-intensity projection (MIP).

\section{MRI in vivo}

Animal experiments were approved by local governmental authorities. Male Specific Pathogen Free nude mice, aging 4-5 weeks and weighing 18-20 g, were supplied by Department of Experimental Animals, Shanghai Jiaotong University, and maintained under standard housing conditions. All animal experiments were carried out in accordance with guidelines evaluated and approved by the ethics committee of Shanghai Jiaotong University.

YAC-1 (mouse lymphoma cells) tumor bearing a group of nude mice was established by subcutaneous injection of $2 \times 10^{6}$ cells suspended in $200 \mu \mathrm{L}$ PBS. When the diameter of tumor was around $8-10 \mathrm{~mm}$, the group of nude mice was subcutaneously injected in tumor region by ZnO:Gd@HA nanospheres (0.2 mL, 0.2 $\mathrm{mg} / \mathrm{mL}$ ), and MR images were collected using small animal Crossed Coil. All MR imaging was performed on a 3.0-T superconductive whole-body scanner (Signa HDXt GE Healthcare), using a dedicated six-channel phased array sensitivity encoding coil for optimized signal reception in the supine position. The conventional gradient system with an amplitude of $40 \mathrm{mT} / \mathrm{m}$ and a slew rate of $150 \mathrm{mT} / \mathrm{m}$ per millisecond. For interstitial MR imaging, a fast $T_{1}$-weighted $3 \mathrm{D}$ gradient-echo sequence (LAVA) in the coronal plane was acquired after gadopentetate dimeglumine injection. The sequence parameters were TR/TE, 4.6/2.4 ms; flip angle, $12^{\circ}$; FOV, $26 \mathrm{~cm}$; matrix, $320 \times 256$; effective slice thickness, $0.6 \mathrm{~mm}$. The 3D MRIs were then reconstructed from the original data set using maximum-intensity projection.

\section{Cell viability experiments}

HCT116 cells $\left(5 \times 10^{3}\right.$ cells per well) in log phase were incubated into 96-well flat-bottomed plates. The cells were incubated for $24 \mathrm{~h}$ at $37{ }^{\circ} \mathrm{C}$ under $5 \% \mathrm{CO}_{2}$.
The obtained ZnO:Gd@HA nanospheres at concentrations of $0,50,100,200,400$ and $500 \mu \mathrm{g} / \mathrm{mL}$ in PBS were added to the wells of the treatment group, respectively. The cells were incubated for $12 \mathrm{~h}$ at $37{ }^{\circ} \mathrm{C}$ under $5 \% \mathrm{CO}_{2}$. Then, sample was removed from the medium and 10\% CCK/ PBS solution was added to each well and incubated at $37{ }^{\circ} \mathrm{C}$ for $4 \mathrm{~h}$. The amount of cell proliferation was measured by reading the optical density value at $450 \mathrm{~nm}$ using a plate reader. The following formula was used to calculate the viability of cell growth: Viability $(\%)=$ (mean of absorbance value of treatment group/mean absorbance value of control) $X$ $100 \%$. The results were expressed as an average over five nominally identical measurement.

\section{Results and Discussion}

The HA conjugated ZnO:Gd@HA nanospheres are synthesized by conjugating activated HA with surface-aminolated ZnO:Gd@silica nanoparticles through EDC/NHS coupling chemistry. The whole procedure isschematically illustrated in Figure 1. During the reaction, ZnO:Gd quantum dots are prepared (step a) and encapsulated in surface-aminolated amino silica shell (step b). Then, the surface-aminolated ZnO:Gd@silica nanoparticles are grafted by the EDC/NHS-activated HA (step c). Thus, the multifunctional ZnO:Gd@HA nanospheres could be obtained.

The XRD pattern of the as synthesized products is shown in Figure 2. All of diffraction peaks could be indexed to $\mathrm{ZnO}$ with hexagonal wurtzite structure (P63mc space group and JCPDS No. 80-0075). Furthermore, the peaks, assigned to crystalline Gd, Gd oxide, Zn-Gd alloys, or Zn-Gd-O particles in the XRD, could not be found, which reveals that Gd-doping do not change the wurtzite structure of $\mathrm{ZnO}$. However, different to the standard data, the relative intensity of the (002) plane decreases distinctly, implying that the Gd-doping could distort the host lattice. ${ }^{[16]}$ When $\mathrm{ZnO}$ :Gd was coated by silica shells and further surface was conjugated by HA, the peaks are similar to those of $\mathrm{ZnO}: \mathrm{Gd}$ products ex cept to the decreasing intensity and an new amorphous peak at about $20^{\circ}$ due to the formation of amorphous species. The above results reveal successful preparation 


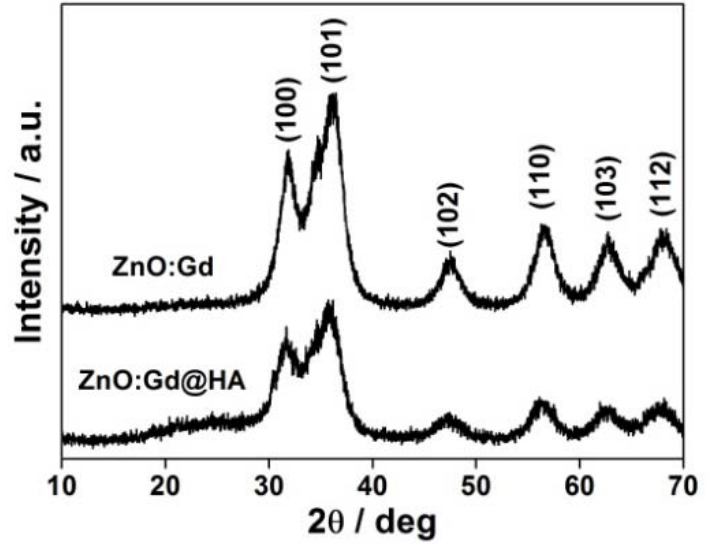

Figure 2 The XRD pattern of obtained ZnO:Gd and ZnO:Gd@HA products.

of ZnO:Gd and their modify by HA.

The morphology of the obtained $\mathrm{ZnO}: \mathrm{Gd}$ products was characterized. Typical TEM image is shown in Figure 3a. It is clear that the nanoparticles are essentially mono-dispersed. HRTEM image reveals the successful preparation of $\mathrm{ZnO}$ :Gd quantum dots with the size about $4 \mathrm{~nm}$ (Figure 3b). Furthermore, the corresponding particle core size histogram is shown in Figure 3c. The result demonstrates that the majority of particle size is $3.8 \mathrm{~nm}$ (the standard deviation was around 7.8\%) for $\mathrm{ZnO}: \mathrm{Gd}$ quantum dots. The result was further proved by UV-vis absorption spectra. As shown in Figure 3d, the ZnO:Gd quantum dots shows a broad absorption band at 325$350 \mathrm{~nm}$. Additionally, their size is evaluated according to Meulenkamp's experiential formula as following: $1240 / \lambda_{1 / 2}=a+b / D^{2}-c / D{ }^{[26]}$ Herein, $\lambda_{1 / 2}$ represents the wavelength where the absorption is half of that at the absorption peak or shoulder; $D$ represents the average size of nanoparticles; $a, b$, and $c$ are parameters when $D$ is within the range $25-65 \AA$. According to the calculation, the particle size is about $4.0 \mathrm{~nm}$, which was in accordance with the result of HRTEM.
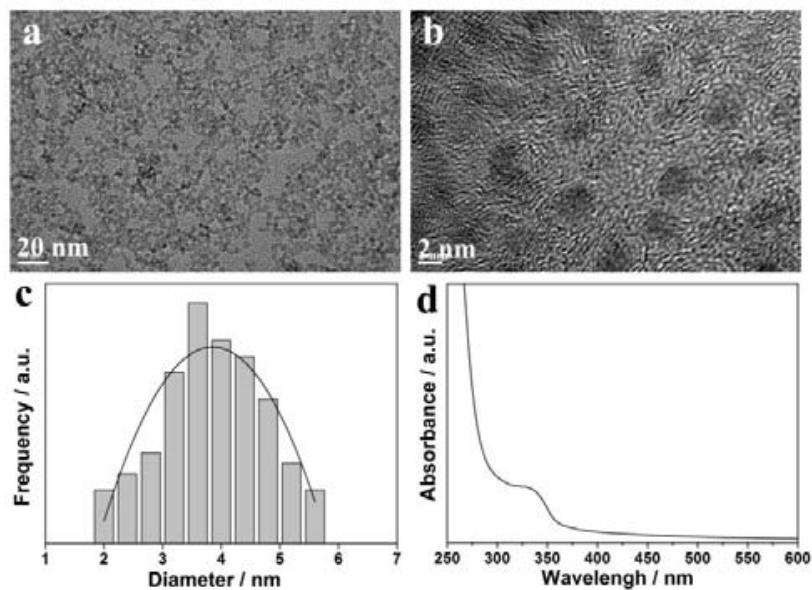

Figure 3 (a) TEM image, (b) HRTEM image, (c) particle core size histogram, and (d) UV-Vis spectra of obtained ZnO:Gd quantum dots.
Furthermore, the $\mathrm{ZnO}: \mathrm{Gd}$ quantum dots were coated by silica shell (ZnO:Gd@silica) and further surface conjugated by HA molecule (ZnO:Gd@HA). As shown in Figure 4a, SEM image shows the obtained products are sphere-like structure. The inserted statistical size analysis of SEM image in Figure 4a reveals that the average diameter of ZnO:Gd@HA nanospheres is 200$300 \mathrm{~nm}$ (the standard deviation was around 4.6\%). Moreover, HRTEM image in Figure 4b indicates these nanospheres contain many nanoparticles inside (highlighted by circles) and thin silica shell outside, implying the successful silica coating. The result implies that the ZnO:Gd@HA nanospheres are built up by primary ZnO:Gd quantum dots. Furthermore, FT-IR spectra of ZnO:Gd@HA nanospheres and HA molecule are shown in Figure 4c. As shown in curve of HA, the typical peaks such as 1610,1560 and $1040 \mathrm{~cm}^{-1}$ are monitored. Among them, the bands at 1610 and $1560 \mathrm{~cm}^{-1}$ are attributed to characteristic stretching vibrations of $\mathrm{C}=\mathrm{O}$ and $\mathrm{C}-\mathrm{N}$, respectively, where the band at $1040 \mathrm{~cm}^{-1}$ is the characteristic bending vibration of $\mathrm{N}-\mathrm{H}$. Compared to curves of HA and ZnO:Gd@HA nanospheres, the main characteristic peaks of HA molecule are found in the spectra of ZnO:Gd@HA nanospheres, such as 1610 and $1040 \mathrm{~cm}^{-1}$, Additionally, some typical peaks about vibrations of $\mathrm{C}-\mathrm{N}$ coming from APS and HA molecules are shown in $1490-1570 \mathrm{~cm}^{-1}$. Moreover, the characteristic peaks of the Si-O-Si symmetrical stretching at about $1100 \mathrm{~cm}^{-1}$, the $\mathrm{Si}-\mathrm{C}$ bending at about 950 $\mathrm{cm}^{-1}$ and the $\mathrm{Si}-\mathrm{C}$ stretching at about $1250 \mathrm{~cm}^{-1}$, are the proof of the silica shells. The results further indicate the silica shell and HA molecule successfully encapsulate some primary $\mathrm{ZnO}: \mathrm{Gd}$ nanoparticles to produce ZnO:Gd@HA nanospheres. Moreover, UV-vis absorption spectrum is performed to evaluate optical property. As shown in Figure 4d, the ZnO:Gd@HA nanospheres show a broad absorption band at 350-400 nm, which is red-shifted by about $40 \mathrm{~nm}$. The reason could lies in the assembly of nanoparticles and surface conjugation of
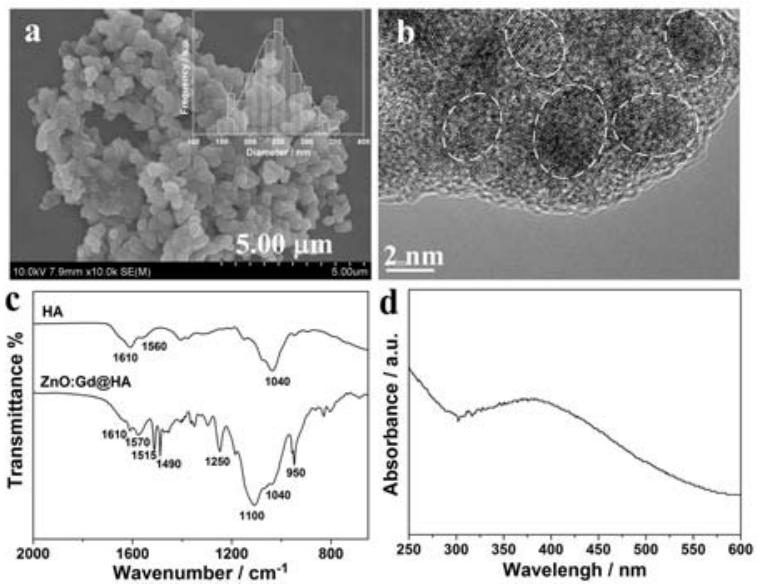

Figure 4 (a) SEM, (b) HRTEM, (c) FT-IR spectra, and (d) UV-vis spectra of obtained ZnO:Gd@HA nanospheres. The inset is the particle core size histogram of ZnO:Gd@HA nanospheres. 


\section{HA molecules.}

The luminescence property of ZnO:Gd@HA nanospheres was investigated to evaluate its multifunctional properties. Figure 5 indicates the room temperature photoluminescent spectra of ZnO:Gd@HA nanospheres. The sample reveals an emission with a maximum centered at $530 \mathrm{~nm}$ due to the near-band-edge emission, which is the green emission component proved by the inserted photoluminescent photograph. The QY of obtained ZnO:Gd@HA nanospheres are measured according to a reported literature, ${ }^{[27]}$ in which the rhodamine 6G ethanol solution $(\mathrm{QY}=95 \%)$ was chose as a standard. Thus, the QY of ZnO:Gd@HA nanospheres is measured and calculated to be $19 \%$.

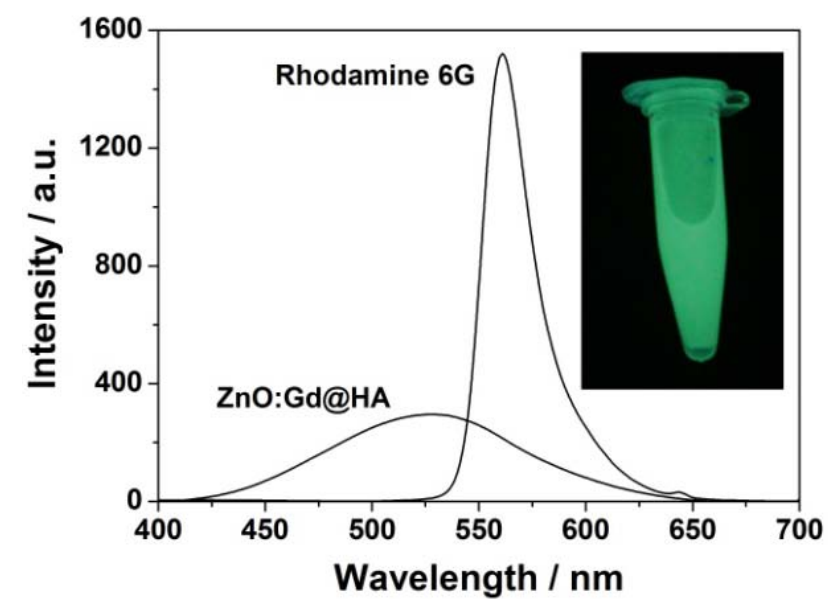

Figure 5 The photoluminescence spectra of ZnO:Gd@HA nanospheres and rhodamine 6G ethanol solution as the standard. The inset is photoluminescent photograph of ZnO:Gd@HA nanospheres.

The magnetic resonance property of ZnO:Gd@HA nanospheres is further investigated. $T_{1}$-weighted $\mathrm{MR}$ images of $\mathrm{ZnO}$ :Gd@HA nanospheres in $\mathrm{H}_{2} \mathrm{O}$ are measured using a 3T MRI system. With increase of the concentration, the ZnO:Gd@HA nanospheres could accelerate longitudianal relaxation of water protons and exert bright contrast (Figure 6a). The resulting $r_{1}$ value is evaluated from a plot of the $T_{1}$ relaxation rate $\left(1 / T_{1}\right)$ versus the $\mathrm{Gd}^{3+}$ ion concentration, and determined to be $5.8 \mathrm{mM}^{-1} \cdot \mathrm{s}^{-1}$, which is larger than that of Magnevist $\left(r_{1}=4.5 \mathrm{mM}^{-1} \cdot \mathrm{s}^{-1}\right) \cdot{ }^{[28]}$ Therefore, these paramagnetic properties can provide ZnO:Gd@HA nanospheres as a good MRI probe.

Furthermore, confocal laser scanning microscopy was used to evaluate the fluorescent imaging of and cellular uptake efficiency of the ZnO:Gd@HA nanospheres. As shown in Figure 7, green fluorescence of the ZnO:Gd@HA nanospheres excited by $405 \mathrm{~nm}$ is shown, which is similar to their results of photoluminescent spectra. Moreover, HCT116 and HEK293 cells with blue fluorescence are dyed with DAPI. In HCT116 cells group, ZnO:Gd@HA nanospheres embrace the cells largely and distribute around them tightly (Figure 6c). However, In HEK293 cells group, ZnO:Gd@HA nano-

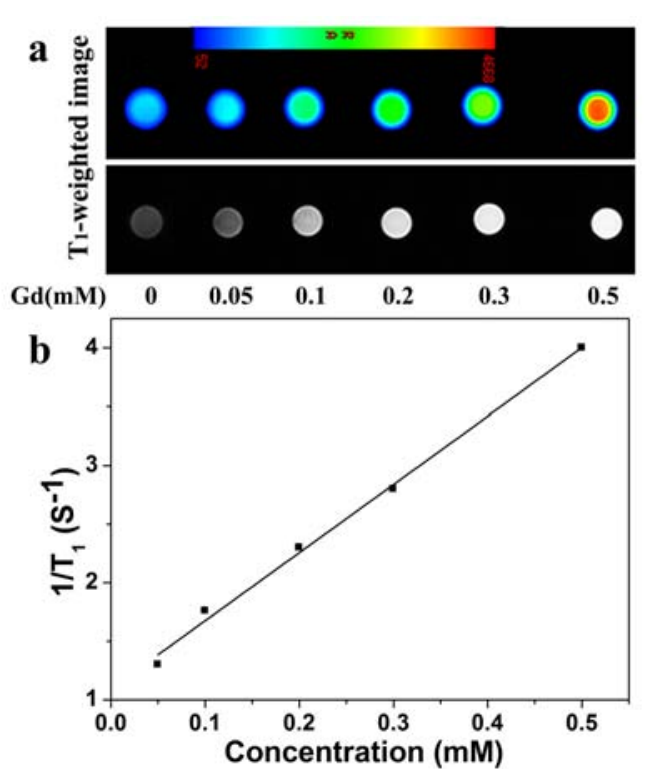

Figure 6 (a) $T_{1}$-weighted maps of various $\mathrm{Gd}^{3+}$ ion concentrations in ZnO:Gd@HA nanospheres and (b) the linear relationship between relaxation rates $\left(1 / T_{1}\right)$ and $\mathrm{Gd}^{3+}$ ion concentrations in ZnO:Gd@HA nanospheres.

spheres reveals poor ability of phagocytosis on cells. As we known, because of the interaction between HA and the CD44 receptor, HA is widely used as a tumor vector for the targeting procedure because CD44 is expressed in a variety of tumors such as breast, colon, intestinal, and brain. Thus, in present experiment, HCT116 cells are used as high CD44 receptor-expressing cells, while HEK293 cells are regarded as control groups because of the low expression of endogenous ligand receptor. The above results imply that the CD44 receptor overexpressed target ability of the obtained HA-based nanoparticles is achieved, which is potential in high targeting efficiency to some kinds of tumors.

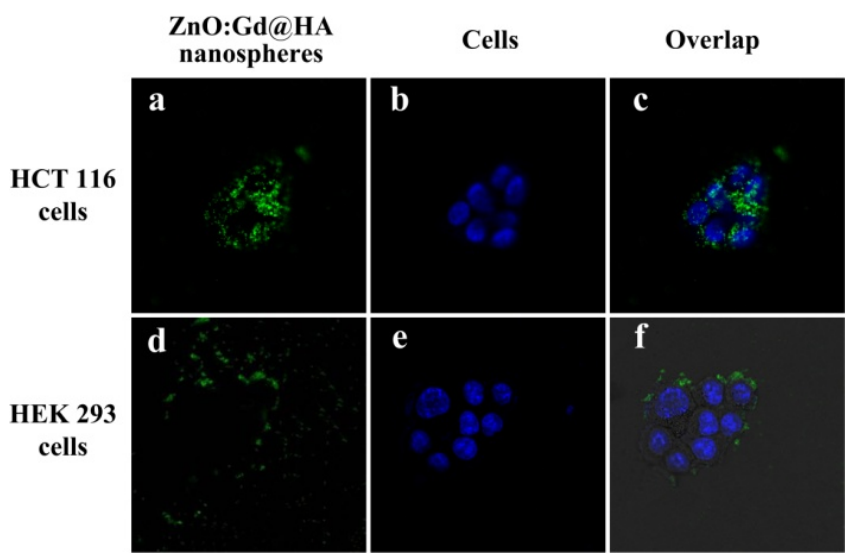

Figure 7 Laser scanning confocal microscopy images of HCT116 and HEK293 cells incubated with ZnO:Gd@HA nanospheres. All images are taken under the identical instrumental conditions and presented at the same intensity scale.

Because of the excellent MRI in vitro and targeting specificity, we further explore the potential to use the 
ZnO:Gd@HA nanospheres for diagnosis of a xenografted tumor model. YAC-1 (mouse lymphoma cells) tumor was used as model because LYVE-1, as a new homologue of the CD44 glycoprotein, is a lymph-specific receptor for HA. Thus, a group of nude mice with about $120 \mathrm{~mm}^{3}$ tumor volume were intratumorally injected with $0.2 \mathrm{~mL}, 0.2 \mathrm{mg} / \mathrm{mL} \mathrm{ZnO}: G d @ H A$ nanospheres. Before and after 5, 15 and 25 min post injection, the nude mice were imaged by MR. As shown in Figure 8, compared to before injection, we can clearly see that the signal of tumor region increases obviously in a typical $T_{1}$-weighted MRI at 5 and $15 \mathrm{~min}$ after injection. Closer observation reveals that some subtle structures in tumor region are also found. However, the brightness of tumor region decreases slightly at 25 min post injection, which could contribute to the metabolism of ZnO:Gd@HA nanospheres. Thus, the intratumoral injection of the ZnO:Gd@HA nanospheres leads to a quite uniform distribution of the particles within the tumor region, allowing for effective and long-periodic MR imaging of the whole tumor, which could come from the high targeting efficiency to the kinds of tumor. Therefore, these result shows that the obtained ZnO:Gd@HA nanospheres have a great potential to be used as a contrast agent for in vivo tumor MR imaging.
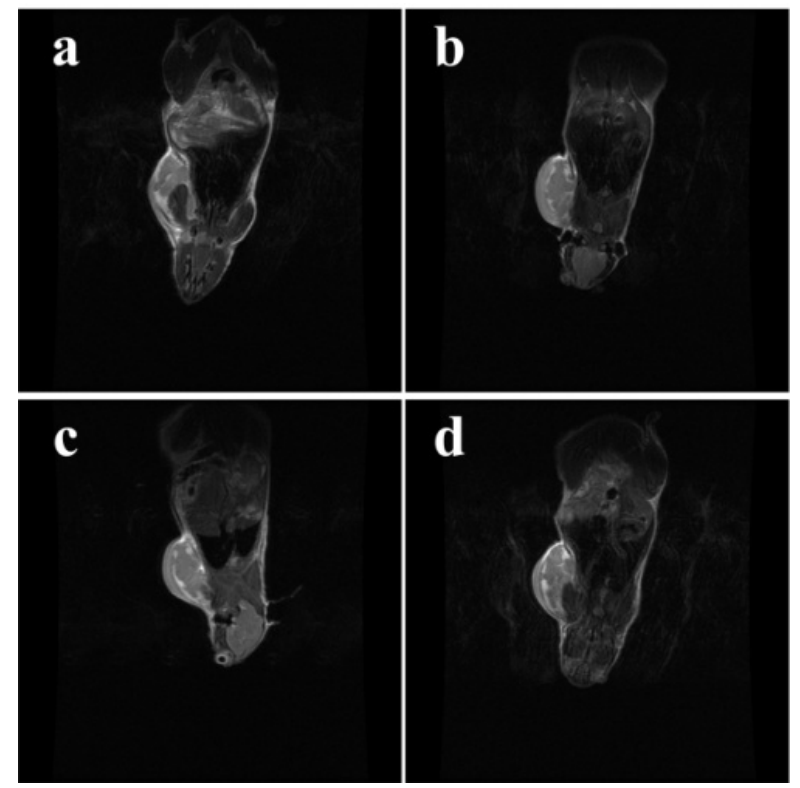

Figure 8 MR images in vivo at different scanning time after post injection. (a) $0 \mathrm{~min}$, (b) $5 \mathrm{~min}$, (c) $15 \mathrm{~min}$ and (d) $25 \mathrm{~min}$.

To examine the feasibility of the obtained ZnO:Gd@ HA nanospheres for use in biomedical applications, their cytotoxicity was investigated. As shown in Figure 9, the effect of different ZnO:Gd@HA nanospheres on cells proliferation was assessed with HCT116 cells by CCK-8 assay. Using the viability of untreated cells as control, the cellular viabilities decrease with an increasing the concentrations of ZnO:Gd@HA nanospheres, and about $80 \%$ cell viabilities are maintained even up to a relatively high dose of $500 \mu \mathrm{g} / \mathrm{mL}$ after exposure for $12 \mathrm{~h}$. In other words, upon incubating with the ZnO:Gd@HA nanospheres of different concentrations, less than $20 \%$ of cells died after $12 \mathrm{~h}$. Therefore, the results demonstrate that the obtained products have a low cytotoxicity.

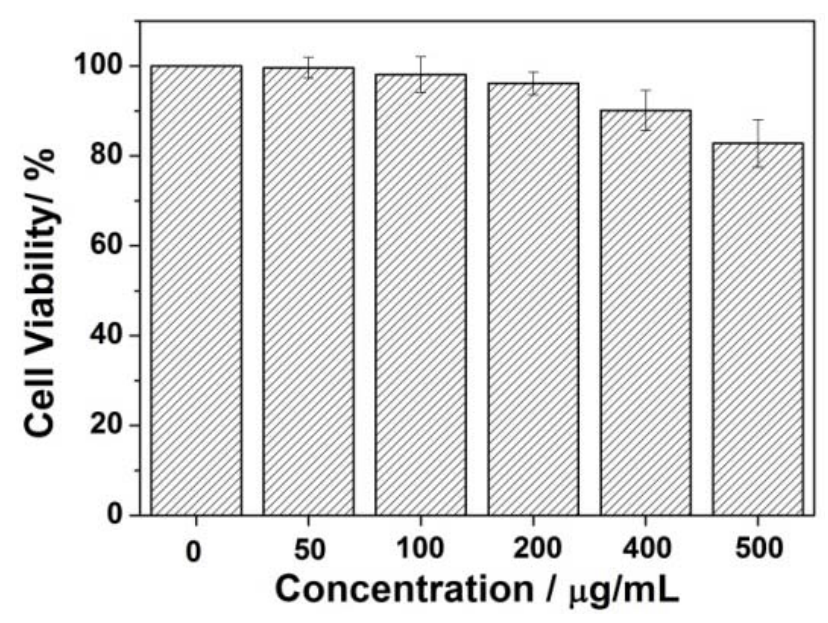

Figure 9 The CCK-8 assay results of the obtained ZnO:Gd@HA nanospheres.

\section{Conclusions}

ZnO:Gd@HA nanospheres are successfully prepared and used as a novel lymphatic system specific multi-modal contrast agent. In vitro characterizations reveal that the ZnO:Gd@HA nanospheres possess suitable and stable physicochemical properties, high quantum yield and relaxation effectiveness and low cytotoxicity. Furthermore, the contrast agent shows an excellent T1-weight signal compared to traditional MRI contrast agents, and significantly higher selectivity to some kinds of lymphoma. Therefore, we believe that the as-synthesized contrast agent is a potential candidate for diagnosis and therapy of lymphatic system.

\section{Acknowledgement}

The work is supported by the Research Project of the Shanghai Municipal Science and Technology Commission (No. 14411968100), the Shanghai Rising-Star Program (No. 13QB1402200) and the Shanghai Minhang District Talent Development Special Fund.

\section{References}

[1] Lauffer, R. B. Chem. Rev. 1999, 99, 2293.

[2] Resch-Genger, U.; Grabolle, M.; Cavaliere-Jaricot, S.; Nitschke, R.; Nann, T. Nat. Methods 2008, 5, 763.

[3] Key, J.; Leary, J. F. Int. J. Nanomed. 2014, 9, 711.

[4] Wang, S. Z.; Jarrett, B. R.; Kauzlarich, S. M.; Louie, A. Y. J. Am. Chem. Soc. 2007, 129, 3848.

[5] Selvan, S. T.; Patra, P. K.; Ang, C. Y.; Ying, J. Y. Angew. Chem., Int. Ed. 2007, 46, 2448.

[6] Su, Y. Y.; He, Y.; Lu, H. T.; Sa, L. M.; Li, Q. N.; Li, W. X.; Wang, L. 
H.; Shen, P. P.; Huang, Q.; Fan, C. H. Biomaterials 2009, 30, 19.

[7] Chen, N.; He, Y.; Su, Y. Y.; Li, X. M.; Huang, Q.; Wang, H. F.; Zhang, X. Z.; Tai, R. Z.; Fan, C. H. Biomaterials 2012, 33, 1238.

[8] Lu, Q.; Wei, D. X.; Cheng, J. J.; Xu, J. R.; Zhu, J. J. Solid State C 2012, 192, 75.

[9] Niu, D. C.; Luo, X. F.; Li, Y. S.; Liu, X. H.; Wang, X.; Shi, J. L. ACS Appl. Mater. Inter. 2013, 5, 9942.

[10] Liu, T.; Liu, X. W.; Yao, Y. J.; Zhou, J.; Zhu, J.; Sun, G.; He, D. N. RSC Adv. 2015, 5, 24049.

[11] Zhang, H. J.; Xiong, H. M. Curr. Mol. Imaging 2013, 2, 177.

[12] Zhao, L. H.; Zhang, J.; Sun, S. Q. J. Lumin. 2012, 132, 2595.

[13] Yin, Q.; Jin, X. Y.; Yang, G. C.; Jiang, C. H.; Song, Z. K.; Sun, G. Y. RSC Adv. 2014, 4, 53561.

[14] Wei, X. J.; Wang, W.; Chen, K. Z. J. Phys. Chem. C 2013, 117, 23716.

[15] Liu, Y. L.; Ai, K. L.; Yuan, Q. H.; Lu, L. H. Biomaterials 2011, 32, 1185.

[16] Li, H. L.; Zhang, Z.; Huang, J. Z.; Liu, R. X.; Wang, Q. B. J. Alloy Compd. 2013, 550, 526.

[17] Saokar, A.; Braschi, M.; Harisinghani, M. Abdom Imaging 2006, 31, 660.
[18] Yan, G. P.; Xu, W.; Yang, L.; Li, L.; Liu, F.; Guo, Q. Z. Pharm. Res. 2010, 27, 1884.

[19] Azoulay, R.; Olivier, P.; Baud, O.; Verney, C.; Santus, R.; Robert, P.; Gressens, P.; Sebag, G. J. Magn. Reson. Imaging 2008, 28, 1046.

[20] Kobayashi, H.; Kawamoto, S.; Bernardo, M.; Brechbiel, M. W.; Knopp, M. V.; Choyke, P. L. J. Control. Release 2006, 111, 343.

[21] Triantafyllou, M.; Studer, U. E.; Birkhäuser, F. D.; Fleischmann, A.; Bains, L. J.; Petralia, G.; Christe, A.; Froehlich, J. M.; Thoeny, H. C. Eur. J. Cancer 2013, 49, 616.

[22] Wu, G. Y.; Zhang, H. J.; Zhan, Z. F.; Lu, Q.; Cheng, J. J.; Xu, J. R.; Zhu, J. Chin. J. Chem. 2015, 33, 1153.

[23] Qiao, Y.; Cheng, J. J.; Wu, G. Y.; Lu, Q.; Yi, F.; Xu, J. R.; Zhu, J.; He D. N. Gen. Chem. 2015, 1, 15.

[24] Banerji, S.; Ni, J.; Wang, S. X.; Clasper, S.; Su, J.; Tammi, R.; Jones, M.; Jackson, D. G. J. Cell Biol. 1999, 144, 789.

[25] Zhang, H. J.; Xiong, H. M.; Ren, Q. G.; Xia, Y. Y.; Kong, J. L. J. Mater. Chem. 2012, 22, 13159.

[26] Meulenkamp, E. A. J. Phys. Chem. B 1998, 102, 7764.

[27] Demasa, J. N.; Crosby, G. A. J. Phys. Chem. 1971, 75, 991.

[28] Sasaki, M.; Shibata, E.; Kanbara, Y.; Ehara, S. Magen. Reson. Med. Sci. 2005, 4, 145. 